\title{
The mini-Bentall approach: Comparison with full sternotomy
}

\author{
Vishal N. Shah, DO, ${ }^{\mathrm{a}}$ Maxwell F. Kilcoyne, DO, ${ }^{\mathrm{b}}$ Meghan Buckley, MS, ${ }^{\mathrm{b}}$ Serge Sicouri, MD, ${ }^{\mathrm{b}}$ and \\ Konstadinos A. Plestis, MD
}

\begin{abstract}
Background: The upper mini sternotomy Bentall (mini-Bentall) procedure may result in less trauma and earlier recovery compared with the full sternotomy Bentall procedure (full Bentall). This study compares immediate and 1- and 3-year survival rates after mini- and full Bentall procedures.
\end{abstract}

Methods: Between February 2009 and July 2019, 48 patients underwent a miniBentall and 49 underwent a full Bentall. Patients who required concomitant procedures, reoperations, or hypothermic circulatory arrest were excluded from our analysis. The mean patient age was 60.7 years in the mini-Bentall group and 59.0 years in the full Bentall group.

Results: There were no in-hospital mortalities. The median cardiopulmonary bypass time (mini-Bentall: 165 minutes [interquartile range (IQR), 155.5-183 minutes]; full Bentall: 164 minutes [IQR, 150-187 minutes]; $P=.619$ ) and aortic cross-clamp times (139 minutes [IQR, 128.5-153 minutes] vs 137 minutes [IQR, 125-156 minutes]; $P=.948)$ were not significantly different between the 2 groups. The mini-Bentall group had a significantly shorter median ventilation time compared with the full Bentall group (5.5 hours [IQR, 3-14 hours] vs 17 hours [IQR, 11-23 hours]; $P<.001)$. None of the patients in the mini-Bentall group had postoperative bleeding necessitating reoperation, whereas 4 patients $(8.2 \%)$ underwent reoperation after full Bentall $(P=.043)$. The mini-Bentall group also had a shorter median hospital length of stay ( 6 days [IQR, $5-8$ days] vs 7 days $[\mathrm{IQR}, 6-8$ days]; $P=.086$ ). Survival at 1 and 3 years was $100 \%$ in both cohorts.

Conclusions: Patients required significantly less ventilation time and reoperations for bleeding after the mini-Bentall procedure. There were no significant differences in cardiopulmonary bypass, aortic cross-clamp times, or intensive care unit and hospital length of stay between the mini-Bentall and full Bentall groups. The miniBentall approach is associated with low morbidity and mortality. (JTCVS Techniques 2021;7:59-66)

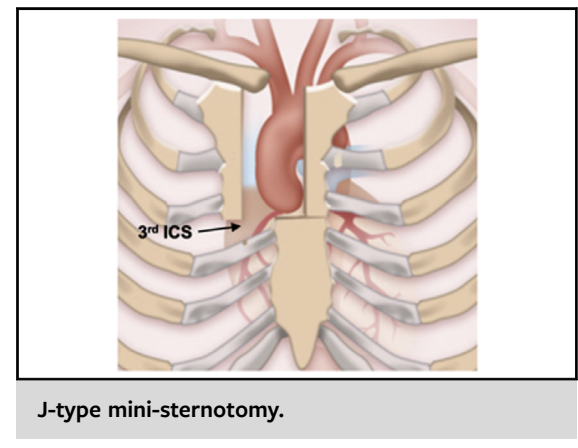

CENTRAL MESSAGE

The mini-Bentall approach is a safe and reproducible technique for aortic root replacement.

\section{PERSPECTIVE}

The low mortality and morbidity after miniBentall, as well as shorter ventilation time and fewer reoperations for bleeding, support its use in experienced hands.

See Commentaries on pages 67 and 69.

\footnotetext{
From the a Department of Cardiothoracic Surgery, University of Nebraska Medical Center, Omaha, Neb; ${ }^{\mathrm{b}}$ Lankenau Institute for Medical Research, Wynnewood, $\mathrm{Pa}$; and ${ }^{\mathrm{c}}$ Department of Cardiothoracic Surgery, Thomas Jefferson University Hospital, Philadelphia, Pa.

Read at the 19th Annual Scientific Meeting of the International Society for Minimally Invasive Cardiothoracic Surgery, New York, NY, May 29 to June 1, 2019.

Received for publication Jan 20, 2021; accepted for publication Jan 20, 2021; available ahead of print Jan 27, 2021.

Address for reprints: Vishal N. Shah, DO, Department of Cardiothoracic Surgery, University of Nebraska Medical Center, 42nd and Emile St, Omaha, NE 68198 (E-mail: ShahVN33@gmail.com).

2666-2507

Copyright (C) 2021 The Authors. Published by Elsevier Inc. on behalf of The American Association for Thoracic Surgery. This is an open access article under the CC BY-NCND license (http://creativecommons.org/licenses/by-nc-nd/4.0/).

https://doi.org/10.1016/j.xjtc.2021.01.025
}

$\rightarrow$ Video clip is available online.

Aortic root replacement (ARR) with composite valve graft (the Bentall procedure) is used to treat ascending aorta and aortic root pathology with good early and long-term results. ${ }^{1,2}$ The procedure is traditionally performed using a full sternotomy (FS; full Bentall) to provide excellent exposure to the ascending aorta, aortic root, and heart. However, an approach to ARR using an upper mini-sternotomy (UMS) could offer less need for blood transfusions, reduced pain, improved respiratory function, and shorter hospital 


$$
\begin{aligned}
& \text { Abbreviations and Acronyms } \\
& \text { ACC }=\text { aortic cross-clamp } \\
& \text { ARR }=\text { aortic root replacement } \\
& \text { CI }=\text { confidence interval } \\
& \text { CPB }=\text { cardiopulmonary bypass } \\
& \text { FS }=\text { full sternotomy } \\
& \text { ICS }=\text { intercostal space } \\
& \text { ICU }=\text { intensive care unit } \\
& \text { IQR }=\text { interquartile range } \\
& \text { LOS }=\text { length of stay } \\
& \text { PVS }=\text { prolonged ventilatory support } \\
& \text { TEE }=\text { transesophageal echocardiography } \\
& \text { UMS }=\text { upper mini-sternotomy }
\end{aligned}
$$

length of stay (LOS) compared with its FS counterpart without compromising procedural exposure. ${ }^{3-7}$ In a previous smaller series, we suggested that the UMS Bentall procedure (ie, mini-Bentall) is safe and reproducible. ${ }^{8}$ With an updated experience over the past 10 years with 48 mini-Bentall and 49 full Bentall procedures, we performed the present study to compare postoperative outcomes between the 2 groups and report short-term followup results.

\section{METHODS \\ Selection Criteria}

Between February 2009 and July 2019, a total of 97 consecutive patients underwent an isolated elective Bentall procedure performed by a single surgeon (K.P.) (Table 1). Forty-eight patients underwent the mini-Bentall procedure and 49 underwent the full Bentall procedure. Isolated elective full Bentall procedures were performed between February 2009 and December 2014, and isolated elective mini-Bentall procedures were performed between May 2015 and July 2019 (Figure 1). Since May 2015, all isolated elective Bentall operations were performed as mini-Bentalls, with no patients requiring a full Bentall. Patients who had a concomitant cardiac procedure or who required open distal anastomosis, hemiarch or total aortic arch replacement using hypothermic circulatory arrest, or repair of type A aortic dissection, reoperation, or root abscess were excluded. Patients were identified from a prospectively maintained aortic registry containing demographic, clinical, operative, and follow-up data, and their data were studied retrospectively. Mortality was assessed using an internal database repository, direct patient contact, query of the Social Security Death Index, and screening of online obituaries. Approvals from the Institutional Review Board for the aortic registry (F/N-R15-3532BLP) and the study (F/N-R163634BLP) were obtained on April 15, 2016. The need for informed consent was waived owing to the study's retrospective nature.

\section{Surgical Technique}

All mini-Bentall patients underwent preoperative computed tomography angiography, with particular attention given to the locations of the aortic annulus and right internal mammary artery. The incision terminates one intercostal space (ICS) above the plane of the aortic annulus, corresponding to the third or fourth ICS. External defibrillation pads were placed because of the limited access within the pericardial space. Standard skin preparation and draping were performed. Subsequently, a J-type ministernotomy was made from $2 \mathrm{~cm}$ inferior to the sternal notch to the midpoint of the third or fourth ICS and exiting the right third or fourth
ICS was done (Figure 2). Particular care was taken during extension of the incision to the corresponding third or fourth ICS to avoid injury to the right internal mammary artery. In 49 patients, a standard FS was performed. In mini-Bentall operations, pericardial traction sutures were used to position the aorta to the midline and close to the sternum. The proximal aortic arch opposite to the innominate artery was directly cannulated using the Seldinger technique under transesophageal echocardiography (TEE) guidance. The right femoral vein was directly cannulated, and a $25 \mathrm{~F}$ long femoral venous cannula (Bio-Medicus; Medtronic, Minneapolis, Minn) was advanced into the superior vena cava using the Seldinger technique under TEE guidance. The femoral venous cannula was connected to a "Y" connector, which had an extra tube attached for additional drainage if needed. After the aorta was cross-clamped (Figure 3, A), 2 L of CustodiolHTK cardioplegia (Essential Pharmaceuticals, Durham, NC) was infused antegradely in the aortic root or directly in the coronary ostia in cases of significant aortic valve regurgitation in all mini-Bentall and in $44.9 \%$ of full Bentall cases. We now use Custodiol-HTK cardioplegia because it allows for at least 1.5 hours of myocardial protection in a single dose. ${ }^{9} \mathrm{~A}$ direct main pulmonary arterial or right superior pulmonary vein vent was inserted. $\mathrm{CO}_{2}$ insufflation was used in both groups, to decrease the possibility of air embolism.

During full Bentall, the right atrium was cannulated directly, and conventional potassium-based blood cardioplegia was administered in the majority of cases $(55.1 \%)$, with repeated doses every 20 minutes. The aorta was transected $1 \mathrm{~cm}$ above the sinotubular junction (Figure $3, B$ ), and attachments of the aortic root to the pulmonary artery and left atrium were sharply divided. Three full-thickness commissural traction stitches were placed (Figure 4, A). The noncoronary sinus was resected, and the right and left main coronary buttons were created. The aortic valve leaflets were excised, and a series of 2-0 Ethibond pledgeted annular sutures were placed to secure the composite valve graft (Figure 4, $B$ ). Either a mechanical or a biological valved conduit was used, based on age and patient preference. The Cor-Knot automated fastener (LSI Solutions, Victor, NY) was used to secure the sutures in all mini-Bentall cases. During the full Bentall, hand-tying was used in 45 patients $(91.8 \%)$ ). The remaining aortic wall was tacked circumferentially to the sewing ring of the composite valve graft with 4-0 polypropylene continuous suture (Video 1). The coronary buttons were anastomosed to the corresponding openings in the graft using thin Teflon felt strip reinforcement. Cardioplegic solution was infused into the graft to check the anastomotic integrity of the coronary buttons to the aortic graft. The ascending aorta was divided approximately $1 \mathrm{~cm}$ proximal to the aortic cross-clamp (ACC). The prosthesis was cut to size, and the distal anastomosis was performed with Teflon felt-reinforced continuous 4-0 polypropylene suture. The posterior aspect of the distal anastomosis was reinforced with interrupted pledgeted 4-0 polypropylene suture. Before securing the distal anastomosis, de-airing maneuvers were performed. The ACC was removed, and the distal anastomosis was secured. The patient was weaned from cardiopulmonary bypass (CPB). TEE was performed to assess for ventricular function and aortic insufficiency. The chest was closed with 3 or 4 stainless steel wires placed around the hemisternum.

\section{Statistical Analysis}

Descriptive statistics for categorical variables are reported as frequency and percentage. The normality of continuous variables was formally tested with the Shapiro-Wilk test and graphically assessed using histograms. Continuous normally distributed data are presented as mean $\pm \mathrm{SD}$, and continuous non-normally distributed data are presented as median and interquartile range (IQR). Categorical distributions were compared between full-Bentall and mini-Bentall groups using the $\chi^{2}$ test, and continuous distributions were compared using the 2-sample $t$ test or Wilcoxon rank-sum test, as appropriate.

An exploratory post hoc analysis was performed to determine whether ventilation time differed between the 2 surgical techniques, after 
TABLE 1. Preoperative characteristics by procedure

\begin{tabular}{|c|c|c|c|c|}
\hline Characteristic & Mini-Bentall $(N=48)$ & Full-Bentall $(\mathrm{N}=49)$ & $P$ value & Overall $(\mathbf{N}=\mathbf{9 7})$ \\
\hline Age, $y$, mean $\pm S D$ & $60.7 \pm 11.7$ & $59.0 \pm 11.7$ & .474 & $59.8 \pm 11.7$ \\
\hline Male sex, n $(\%)$ & $42(87.5)$ & $39(79.6)$ & .294 & $81(83.5)$ \\
\hline Hypertension, $\mathrm{n}(\%)$ & $38(79.2)$ & $38(77.6)$ & .847 & $76(78.4)$ \\
\hline COPD, n $(\%)$ & $5(10.4)$ & $7(14.3)$ & .563 & $12(12.4)$ \\
\hline Diabetes, $\mathrm{n}(\%)$ & $4(8.3)$ & $3(6.1)$ & .674 & $7(7.2)$ \\
\hline Prior stroke, n (\%) & $2(4.2)$ & $3(6.1)$ & .663 & $5(5.2)$ \\
\hline Prior TIA, n (\%) & $1(2.1)$ & $3(6.1)$ & .317 & $4(4.1)$ \\
\hline Renal insufficiency, n (\%) & $2(4.2)$ & $0(0.0)$ & .149 & $2(2.1)$ \\
\hline Dialysis, n (\%) & $0(0.0)$ & $0(0.0)$ & - & $0(0.0)$ \\
\hline Liver failure, $\mathrm{n}(\%)$ & $2(4.2)$ & $1(2.0)$ & .545 & $3(3.1)$ \\
\hline Bicuspid aortic valve, $\mathrm{n}(\%)$ & $16(33.3)$ & $14(28.6)$ & .612 & $30(30.9)$ \\
\hline Chronic dissection, $\mathrm{n}(\%)$ & $0(0.0)$ & $1(2.0)$ & .320 & $1(1.0)$ \\
\hline Medial degeneration, $\mathrm{n}(\%)$ & $48(100.0)$ & $46(93.9)$ & .082 & $94(96.9)$ \\
\hline Infection, $\mathrm{n}(\%)$ & $0(0.0)$ & $0(0.0)$ & - & $0(0.0)$ \\
\hline Pseudoaneurysm, n (\%) & $1(2.1)$ & $1(2.0)$ & .988 & $2(2.1)$ \\
\hline Aortitis, n (\%) & $0(0.0)$ & $1(2.0)$ & .320 & $1(1.0)$ \\
\hline
\end{tabular}

$S D$, Standard deviation; $C O P D$, chronic obstructive pulmonary disease; $T I A$, transient ischemic attack

controlling for possible confounding variables. The relationship was tested using a multivariable negative binomial regression model, as ventilation time was highly right-skewed, and surgical technique covariate adjusted regression coefficient $\left(\widehat{B}_{\text {full Bentall }}\right)$ and $95 \%$ confidence interval $(\mathrm{CI})$ are presented. Age, sex, history of prior transient ischemic attack, history of liver failure, packed red blood cell units, and cryoprecipitate units were all chosen as potential confounding variables, as they changed surgical technique's regression coefficient by $\geq 10 \%$ in bivariate models.

One-year and 3-year survival estimates and $95 \%$ CIs were obtained from Kaplan-Meier survival curves. The log-rank test could not be used to compare the survival distributions between the mini-Bentall and full Bentall groups, because there were no deaths in either group before 3.7 years, which was the longest duration of follow-up for the miniBentall group. All statistical tests were 2-sided, with the $\alpha$ level set at 0.05 for statistical significance. All analyses were conducted using Stata/ MP 15.1 (StataCorp, College Station, Tex).

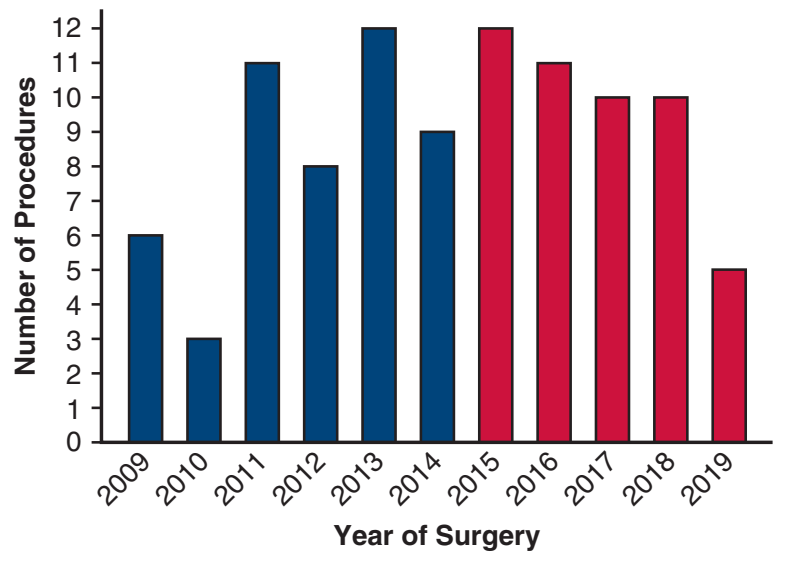

Full Bentall $\square$ Mini Bentall

FIGURE 1. Distribution by year of isolated elective mini-Bentall and full Bentall procedures performed by a single surgeon.

\section{Data Analysis}

The primary outcomes were in-hospital mortality and 1- and 3-year survival rates. The follow-up interval was calculated as the time from the initial operation until the last documented follow-up visit or the date of death. Secondary outcomes included stroke, renal insufficiency, prolonged ventilatory support (PVS), reoperation for bleeding, CPB and ACC times, and intensive care unit (ICU) and hospital LOS. Stroke was defined as a new neurologic deficit that persisted at discharge, accompanied by imaging findings confirming a new brain injury. Postoperative renal insufficiency was defined as doubling of the serum creatinine or a new requirement for hemodialysis. PVS was defined as the need for $>24$ hours of mechanical

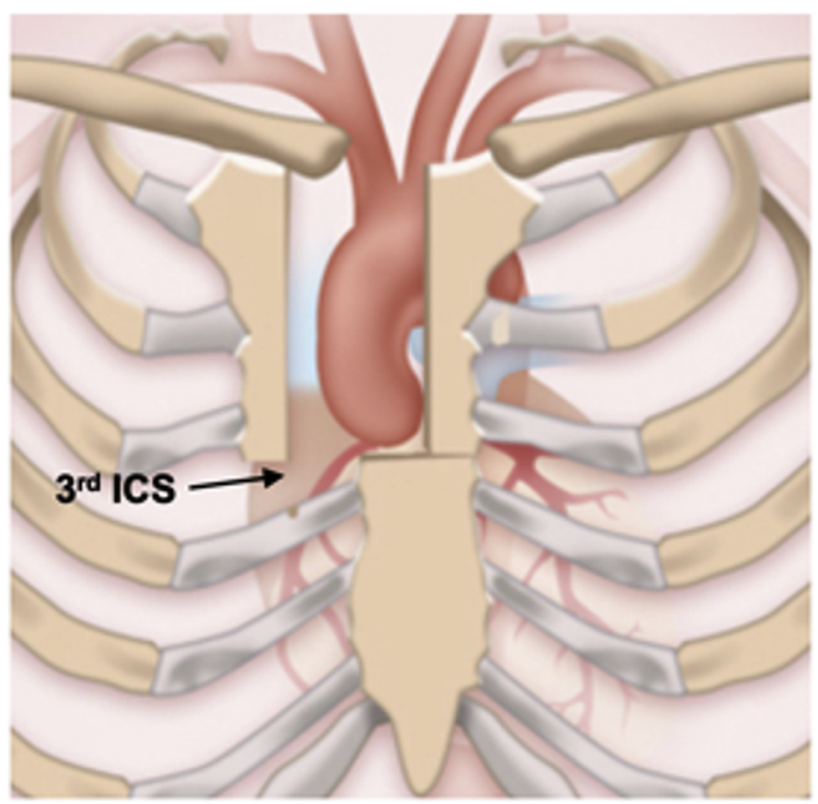

FIGURE 2. J-type mini-sternotomy. ICS, Intercostal space. 

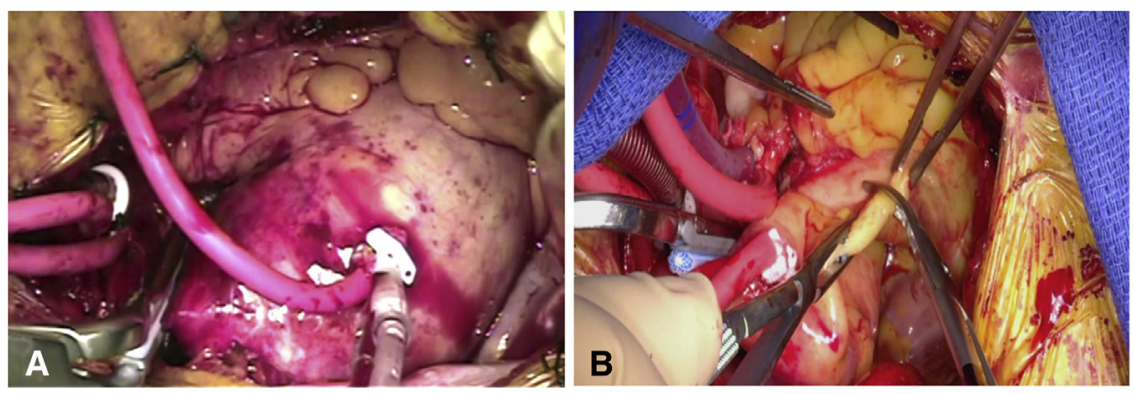

FIGURE 3. Exposure of the aortic root (A) and transverse aortotomy $1 \mathrm{~cm}$ above the sinotubular junction (B).

ventilation postoperatively. Other complications were defined according to the Society of Thoracic Surgeons Adult Cardiac Surgery Database specifications. The authors' blood transfusion and extubation protocols and ICU and hospital discharge criteria are provided in Appendix 1.

\section{RESULTS}

Baseline demographic data are presented in Table 1. Forty-eight patients underwent the mini-Bentall procedure, and 49 patients underwent the full Bentall procedure. There were no significant differences in any of the baseline demographics between the mini-Bentall and full-Bentall groups $(P>.05$ for all). There was a male preponderance in both groups, and the mean patient age was $60.7 \pm 11.7$ years in the mini-Bentall group and $59.0 \pm 11.7$ years in the full Bentall group. Hypertension $(79.2 \%$ vs $77.6 \%)$ and chronic obstructive pulmonary disease $(10.4 \%$ vs $14.3 \%)$ were the 2 most frequent comorbidities in the miniBentall and full-Bentall groups. Aortic pathology in the 2 groups included medial degeneration (48 patients [100\%] vs 46 patients $[93.9 \%] ; P=.082)$ and chronic dissection $(0 \%$ vs $2.0 \% ; P=.320)$. Bicuspid aortic valves were seen in $33.3 \%$ of the mini-Bentall patients and in $28.6 \%$ of the full Bentall patients.

There were no in-hospital deaths in either group. One stroke $(2.1 \%)$ occurred in the mini-Bentall group. The patient was a 55-year-old male who developed multiple cerebellar and occipital emboli and had a severely atherosclerotic distal arch and proximal descending aorta. He had upper extremity weakness postoperatively that had nearly resolved before discharge. The median CPB time was similar in the mini-Bentall and full Bentall groups (165 minutes [IQR, 155.5-183 minutes] vs 164 minutes [IQR, 150-187 minutes]; $P=.619$ ) (Table 2). Similarly, the median ACC time was not significantly different in the 2 groups (139 minutes [IQR, 128.5-153 minutes] vs 137 minutes [IQR, 125-156 minutes]; $P=.948)$. The mini-Bentall group had a significantly shorter median ventilation time (5.5 hours [IQR, 3-14 hours] vs 17 hours [IQR, 11-23 hours]; $P<.001)$. This difference remained significant after controlling for age, sex, history of prior transient ischemic attack, history of liver failure, packed red blood cell units, and cryoprecipitate units $\left(\widehat{B}_{\text {full Bentall }}=0.90\right.$; 95\% CI, 0.52-1.28; $P<.001)$. There were marginal differences in PVS between the mini-Bentall and full-Bentall groups $(8.3 \%$ vs $22.5 \% ; P=.055)$. No postoperative wound infections occurred in either group. There was no significant between-group difference in intraoperative red blood cell transfusion ( 0 vs $0 ; P=.435)$. No conversions to FS were observed during mini-Bentall. None of the patients in the mini-Bentall group had postoperative bleeding necessitating reoperation, whereas 4 patients $(8.2 \%)$ underwent reoperation after full Bentall $(P=.043)$. Other postoperative variables are compared in Table 3 .

The median ICU stay was 3 days for both groups $(P=.743)$. The median LOS was $<6$ days (IQR, 5-8 days) for the mini-Bentall group compared with
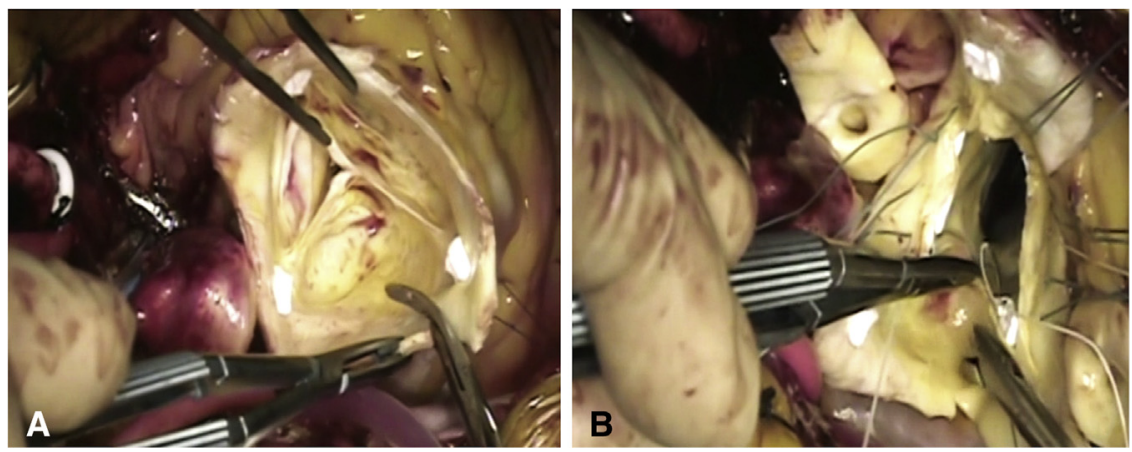

FIGURE 4. Three traction sutures placed at the commissures (A) and placement of annular sutures (B). 
TABLE 2. Intraoperative results by procedure

\begin{tabular}{|c|c|c|c|c|}
\hline Parameter & Mini-Bentall $(N=48)$ & Full Bentall $(N=49)$ & $P$ value & Overall $(\mathbf{N}=97)$ \\
\hline CPB time, min, median (IQR) & $165(155.5-183)$ & $164(150-187)$ & 619 & $165(152-185)$ \\
\hline ACC time, min, median (IQR) & $139(128.5-153)$ & $137(125-156)$ & .948 & $138(128-154)$ \\
\hline RBC, units, median (IQR) & $0(0-1)$ & $0(0-2)$ & .435 & $0(0-1)$ \\
\hline FFP, units, median (IQR) & $0(0-2)$ & $0(0-2)$ & .395 & $0(0-2)$ \\
\hline Cryoprecipitate, units, median (IQR) & $0.5(0-2)$ & $0(0-0)$ & .003 & $0(0-2)$ \\
\hline Platelets, units, median (IQR) & $1(0-2)$ & $0(0-2)$ & .429 & $1(0-2)$ \\
\hline Custodiol-HTK cardioplegia, n (\%) & $48(100.0)$ & $22(44.9)$ & $<.001$ & $70(72.2)$ \\
\hline Conventional potassium-based blood cardioplegia, n (\%) & $0(0.0)$ & $27(55.1)$ & $<.001$ & $27(27.8)$ \\
\hline Cor-Knot fastener, n (\%) & $48(100.0)$ & $4(8.2)$ & $<.001$ & $52(53.6)$ \\
\hline
\end{tabular}

Significant $P$ values are in bold type. $C P B$, Cardiopulmonary bypass; $I Q R$, interquartile range; $A C C$, aortic cross-clamp; $R B C$, red blood cells; $F F P$, fresh frozen plasma.

7 days (IQR, 6-8 days) for the full Bentall group $(P=.086)$. For the whole cohort, survival at both 1 years and 3 years was $100 \%(95 \% \mathrm{CI}$, not applicable; no deaths). There were no observed deaths in the follow-up period for the mini-Bentall patients (maximum follow-up time, 3.7 years), and 2 deaths in the full Bentall group, occurring at 4.1 and 5.5 years postoperatively (maximum follow-up time, 9.7 years).

\section{DISCUSSION}

Two recent large meta-analyses comparing minimally invasive surgery aortic root surgery with FS reported reduced ICU and hospital LOS and shorter CPB time after minimally invasive surgery. However, these studies had significant heterogeneity related to procedure types, patient and study characteristics, incision types, and study periods. ${ }^{7,10}$ To further complicate matters, the few studies focusing solely on Bentall procedures are hampered by less recent data, absence of an FS control group, or small numbers of patients. ${ }^{3,11,12}$ To compare, we report postoperative outcomes in a contemporary cohort of 97 patients with similar baseline characteristics undergoing UMS and FS Bentall procedures performed by a single surgeon. We found no significant differences in CPB times or ICU and hospital LOS between the 2 groups, but did observe less ventilation time and fewer reoperations in the miniBentall group.

Our overall hospital mortality was zero, in line that reported by Mikus and colleagues $(0 \%),{ }^{3}$ Wachter and colleagues $(0.9 \%),{ }^{4}$ and Shrestha and colleagues $(0 \%){ }^{13}$ In our total cohort, the incidence of stroke $(1.0 \%)$ was lower than or comparable to that of other studies $(0 \%$ $5.0 \%)^{5,10,14}$ No patient experienced postoperative renal failure or sternal infection. The most common complication observed in both groups was atrial fibrillation $(27.8 \%$ overall). Despite minimal manipulation of the heart, atrial fibrillation is still a significant complication after mini-Bentall, as corroborated by Perrotta and colleagues,${ }^{11}$ who reported an atrial fibrillation rate of $22.5 \%$. The mini-Bentall patients had a shorter hospital LOS (6 days vs 7 days; $P=.086$ ), although the difference was not statistically significant. Other studies comparing LOS after UMS and FS for ascending aorta and hemiarch surgery (5 days vs 6 days; $P=.002)$ and aortic root surgery (6.8 days vs 7.5 days; $P=.03$ ) revealed similar findings. ${ }^{5,7}$ Our median ICU LOS (3 days for both groups) was comparable to

TABLE 3. Postoperative results by procedure

\begin{tabular}{|c|c|c|c|c|}
\hline Parameter & Mini-Bentall $(N=48)$ & Full Bentall $(N=49)$ & $P$ value & Overall $(\mathbf{N}=97)$ \\
\hline Hospital mortality, n (\%) & $0(0.0)$ & $0(0.0)$ & - & $0(0.0)$ \\
\hline ICU LOS, d, median (IQR) & $3(2-5)$ & $3(2-4)$ & .743 & $3(2-4)$ \\
\hline Hospital LOS, d, median (IQR) & $6(5-8)$ & $7(6-8)$ & .086 & $6(5-8)$ \\
\hline Stroke, n (\%) & $1(2.1)$ & $0(0.0)$ & .310 & $1(1.0)$ \\
\hline TIA, n $(\%)$ & $0(0.0)$ & $0(0.0)$ & - & $0(0.0)$ \\
\hline Ventilation time, $\mathrm{h}$, median (IQR) & $5.5(3-14)$ & $17(11-23)$ & $<.001$ & $12(4-21)$ \\
\hline PVS $(>24$ h), n (\%) & $4(8.3)$ & $11(22.5)$ & .055 & $15(15.5)$ \\
\hline Renal insufficiency, n (\%) & $0(0.0)$ & $0(0.0)$ & 一 & $0(0.0)$ \\
\hline Wound infection, n (\%) & $0(0.0)$ & $0(0.0)$ & - & $0(0.0)$ \\
\hline Reoperation for bleeding, $\mathrm{n}(\%)$ & $0(0.0)$ & $4(8.2)$ & .043 & $4(4.1)$ \\
\hline Atrial fibrillation, $\mathrm{n}(\%)$ & $13(27.1)$ & $14(28.6)$ & .870 & $27(27.8)$ \\
\hline
\end{tabular}

Significant $P$ values are in bold type. ICU, Intensive care unit; $L O S$, length of stay; $I Q R$, interquartile range; TIA, transient ischemic attack; $P V S$, prolonged ventilatory support. 


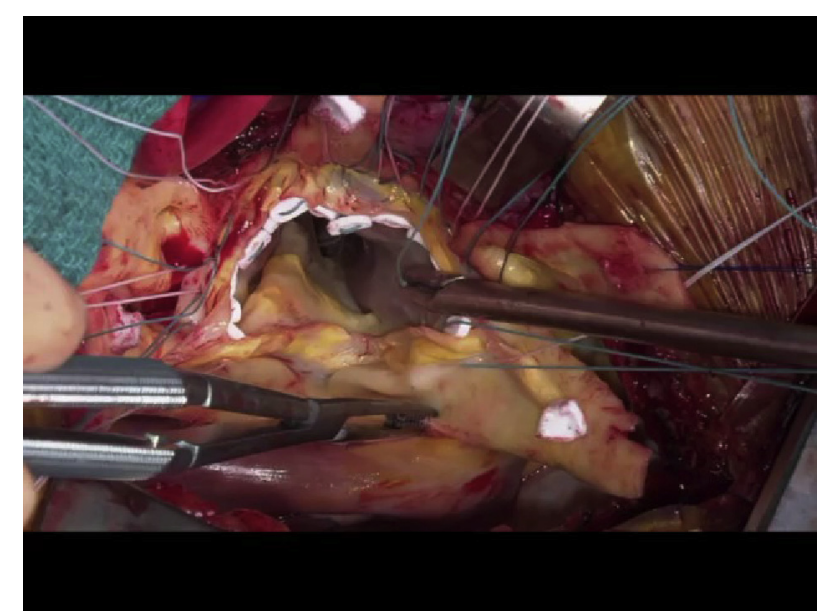

VIDEO 1. The mini-Bentall approach. Video available at: https://www. jtcvs.org/article/S2666-2507(21)00097-3/fulltext.

that reported by Wachter and colleagues ${ }^{4}$ (UMS, $1.9 \pm 3.6$ days vs FS, $3.2 \pm 5.6$ days; $P<.001$ ), Perrotta and colleagues ${ }^{11}$ (UMS, $3.3 \pm 8.2$ days), and Hillebrand and colleagues ${ }^{12}$ (UMS, $2.45 \pm 3.43$ days vs FS, $3.92 \pm 7.53$ days) following valve-sparing ARRs and Bentall procedures, respectively. OS rates at 1 year and 3 years were both $100 \%$, comparable to other studies, in which they ranged from $94.1 \%$ to $100 \%$ and from $80.0 \%$ to $97.2 \%$, respectively. ${ }^{1,2,5,11,14}$

No significant between-group differences in other postoperative outcomes were noted, except for time on the ventilator and reoperations for bleeding. The mini-Bentall group required significantly less time on the ventilator (5.5 hours vs 17 hours; $P<.001$ ), possibly related to preserved respiratory mechanics from less trauma. Likewise, Mikus and colleagues ${ }^{3}$ found significantly fewer patients requiring ventilation for $>96$ hours after mini- Bentall than after full-Bentall $(2 \%$ vs $10 \% ; P<.01)$. Our cohort underwent no reoperations for bleeding after mini-Bentall procedures, but $4(8.2 \%)$ reoperations after full Bentall procedures $(P=.043)$. One patient required reoperation for an epicardial lead abrasion, which required a single pledgeted 4-0 Prolene stitch. No definite sources of bleeding were identified at reoperation and were likely related to coagulopathy in the other patients. Previous studies have reported reoperation rates for bleeding ranging from $1.3 \%$ to $6.0 \%$ for UMS and from $3.1 \%$ to $10.0 \%$ for FS aortic root surgery. ${ }^{3,6,7,13,14}$ The same techniques were used in both groups to avoid reoperation for bleeding, including tacking the remaining aorta to the aortic cuff of the aortic valve, reinforcing the coronary buttons with Teflon felt and the distal anastomosis with pledgeted polypropylene sutures, and assessing the integrity of the coronary buttons by injecting cardioplegia into the aortic root. Opening the right pleura will help avoid tamponade. Previous studies have report
CPB and ACC times ranging from 70 to 166 minutes and from 55 to 131 minutes, respectively, for a variety of UMS ARR procedures. ${ }^{3-7,12}$ Although Mikus and colleagues $^{3}$ reported reductions in CPB time (95.7 minutes vs 112.8 minutes; $P=.004$ ) and ACC time ( 81.5 minutes vs 94.0 minutes; $P=.04$ ) with mini-Bentall, we found no significant between-group differences in these parameters. For the mini-Bentall and full-Bentall groups, CPB time (165 minutes vs 164 minutes; $P=.619$ ) and ACC time (139 minutes vs 137 minutes; $P=.948$ ) were similar despite the use of Cor-Knot and Custodiol-HTK cardioplegia in all mini-Bentall procedures. Although the tandem use of these technologies has been shown to decrease CBP and ACC times in mini-aortic valve replacement, ${ }^{15}$ these results were not borne out in the present study, possibly related to the more time-consuming technical nature of mini-Bentall in our experience.

The UMS approach is advantageous and feasible with thorough planning and execution. The UMS incision affords excellent exposure of the ascending aorta and arch for cannulation and distal anastomosis and avoids dissection and possible injury to the right ventricle, as visualization of the heart is unnecessary. It also permits prompt conversion to FS if needed. Strategically placed pericardial traction sutures bring the aorta to the midline and near the sternum. Horizontal mattress traction sutures are placed above the 3 commissures and secured under tension to the surgical drapes to bring the aortic root near the sternum as well. Another adjunctive technique that improves exposure is placement of cephalad traction sutures around the ACC and through the edge of the pericardium. In patients with deep mediastinal structures, pericardial sutures are placed and tagged, the sternal retractor is removed, upward traction is then placed on the pericardial sutures, and the sternal retractor is reinserted. Central arterial cannulation is done using the Seldinger technique, because direct cannulation may be difficult in a UMS. Femoral venous cannulation using the Seldinger technique under TEE guidance clears the operative field and provides excellent venous drainage. In addition, it is important to expose the right common femoral vein via a small groin incision to avoid inadvertent injury to the common femoral artery, particularly in cases of anatomic variations. In cases of inadequate venous drainage, it is helpful to "Y" the venous line by inserting a second smaller cannula directly into the right atrium.

By using a stepwise progression, surgeons can gain experience in a safe and reproducible manner. Shrestha and colleagues $^{13}$ have highlighted the importance of the stepby-step approach to address the steep learning curve associated with minimally invasive proximal aortic surgery. The surgeon should perform initially a high volume of full Bentalls and simpler surgeries, such as aortic valve 
replacement, using UMS. Only after gaining sufficient experience should the surgeon advance to more complex operations. Excellent UMS candidates are those patients presenting for isolated elective Bentall procedures. Patients presenting with acute type $\mathrm{A}$ dissections, those requiring concomitant coronary artery bypass grafting or total arch replacement, or those with prior coronary artery bypass grafting with patent internal mammary grafts crossing the midline are not candidates for the UMS approach owing to the need for greater operative exposure and higher technical complexity. Patients who require reoperations, simultaneous mitral valve surgery, hemiarch replacement, open distal anastomosis, or have root abscesses may be candidates for UMS; however, these procedures should be performed by surgeons with expertise in the UMS approach.

There are some limitations to this study. It is a retrospective review of a large prospectively maintained aortic registry. All cases were performed by a single surgeon with $>20$ years' experience in aortic surgery, which may limit the generalizability of our findings. Larger, prospective studies are needed to verify our results.

\section{CONCLUSIONS}

Our contemporary Bentall experience over the past decade refers to a series of 48 mini-Bentall and 49 full Bentall procedures. The median ventilation time and reoperation for bleeding was significantly less after mini-Bentall. No other significant differences were noted between the 2 groups. The mini-Bentall approach can be performed safely by adhering to key technical principles.

\section{Conflict of Interest Statement}

The authors reported no conflicts of interest.

The Journal policy requires editors and reviewers to disclose conflicts of interest and to decline handling or reviewing manuscripts for which they may have a conflict of interest. The editors and reviewers of this article have no conflicts of interest.

We thank Donna Loyle, MS, for editorial assistance.

\section{References}

1. Chakos A, Yan TD. Bentall and mini-Bentall procedure. In: Raja SG, ed. Cardiac Surgery: A Complete Guide. 1st ed. Switzerland: Springer; 2020:307-14.

2. Di Marco L, Pacini D, Pantaleo A, Leone A, Barberio G, Marinelli G, et al. Composite valve graft implantation for the treatment of aortic valve and root disease: results in 1045 patients. J Thorac Cardiovasc Surg. 2016;152: 1041-8.e1.

3. Mikus E, Micari A, Calvi S, Salomone M, Panzavolta M, Paris M, et al. MiniBentall: an interesting approach for selected patients. Innovations (Phila). 2017; 12:41-5.

4. Wachter K, Franke UFW, Yadav R, Nagib R, Ursulescu A, Ahad S, et al. Feasibility and clinical outcome after minimally invasive valve-sparing aortic root replacement. Interact Cardiovasc Thorac Surg. 2017;24:377-83.

5. Tabata M, Khalpey Z, Aranki SF, Couper GS, Cohn LH, Shekar PS. Minimal access surgery of ascending and proximal arch of the aorta: a 9-year experience. Ann Thorac Surg. 2007;84:67-72.

6. Levack MM, Aftab M, Roselli EE, Johnston DR, Soltesz EG, Gillinov AM, et al Outcomes of a less-invasive approach for proximal aortic operations. Ann Thorac Surg. 2017;103:533-40.

7. Harky A, Al-Adhami A, Chan JSK, Wong CHM, Bashir M. Minimally invasive versus conventional aortic root replacement-a systematic review and metaanalysis. Heart Lung Circ. 2019;28:1841-51.

8. Abjigitova D, Panagopoulos G, Orlov O, Shah V, Plestis KA. Current trends in aortic root surgery: the mini-Bentall approach. Innovations (Phila). 2018;13: 91-6.

9. Savini C, Murana G, Di Eusanio M, Suarez SM, Jafrancesco G, Castrovinci S, et al. Safety of single-dose histidine-tryptophan-ketoglutarate cardioplegia during minimally invasive mitral valve surgery. Innovations (Phila). 2014;9: 416-20.

10. Rayner TA, Harrison S, Rival P, Mahoney DE, Caputo M, Angelini GD, et al Minimally invasive versus conventional surgery of the ascending aorta and root: a systematic review and meta-analysis. Eur J Cardiothorac Surg. 2020; 57:8-17.

11. Perrotta S, Lentini S, Rinaldi M, D'armini AM, Tancredi F, Raffa G, et al. Treatment of ascending aorta disease with Bentall-De Bono operation using a miniinvasive approach. J Cardiovasc Med (Hagerstown). 2008;9:1016-22.

12. Hillebrand J, Alshakaki M, Martens S, Scherer M. Minimally invasive aortic root replacement with valved conduits through partial upper sternotomy. Thorac Cardiovasc Surg. 2018;66:295-300.

13. Shrestha M, Krueger H, Umminger J, Koigeldiyev N, Beckmann E, Haverich A et al. Minimally invasive valve sparing aortic root replacement (David procedure) is safe. Ann Cardiothorac Surg. 2015;4:148-53.

14. Elghannam M, Aljabery Y, Naraghi H, Moustafine V, Bechte M, Strauch J, et al. Minimally invasive aortic root surgery: midterm results in a 2-year follow-up. $J$ Card Surg. 2020;35:1484-91.

15. Plestis K, Orlov O, Shah VN, Wong J, Thomas M, Aharon A, et al. Facilitating technologies in minimally invasive aortic valve replacement: a propensity score analysis. Interact Cardiovasc Thorac Surg. 2018;27:202-7.

Key Words: mini-Bentall, upper mini-sternotomy, aortic root replacement, full sternotomy 


\section{APPENDIX 1}

\section{Blood Transfusion Protocol}

During the study period, patients received a transfusion at an $\mathrm{Hb} \leq 6$ on $\mathrm{CPB}$. Postoperatively, patients received a transfusion at an $\mathrm{Hb} \leq 8$, particularly if they had signs of hemodynamic instability or active blood loss.

\section{Extubation Protocol}

Our screening test to determine whether patients could be weaned off of mechanical ventilation included oxygen saturation $>90 \%$, with a fraction of inspired oxygen $\left(\mathrm{FiO}_{2}\right)$ $>40 \%$ and a positive end-expiratory pressure of $8 \mathrm{~cm} \mathrm{H}_{2} \mathrm{O}$, and a good level of consciousness with no need for escalating doses of vasoactive and sedative agents. A patient successfully passing this screening test was placed on a 30-minute to 2-hour spontaneous breathing trial on pressure support of $5 \mathrm{~cm} \mathrm{H}_{2} \mathrm{O}$ or continuous positive airway pressure of 5 to $8 \mathrm{~cm} \mathrm{H}_{2} \mathrm{O}$. If the patient did not develop tachypnea or hemodynamic distress, then extubation was done.

\section{ICU Discharge Criteria}

Patients were considered eligible for ICU discharge when they met the following criteria: awake and capable of communication, extubated on nasal cannula with $\mathrm{SpO}_{2}$ $>95 \%$, no uncontrolled or major arrhythmia, hemodynamic stability without need for vasopressors or inotropic agents, no active blood loss requiring blood transfusions, and normal functioning of body systems.

\section{Hospital Discharge Criteria}

Patients were considered eligible for hospital discharge when they met the following criteria: stable hemodynamics with normal pulse and vitals without the need for vasoactive agents, afebrile, no signs of wound infection, normal bowel function, normal laboratory values, satisfactory ECG and chest X-ray, and good oral intake. 